\title{
Large Area Silica Nano Grids by Homogeneous High Resolution Laser Patterning of $\mathrm{SiO}_{\mathrm{x}}$-Films
}

\author{
David KÖHNE, Thomas FRICKE-BEGEMANN, Ruth WEICHENHAIN-SCHRIEVER, Jürgen IHLEMANN \\ Laser-Laboratorium Göttingen, Hans-Adolf-Krebs-Weg 1, 37077 Göttingen, Germany \\ E-mail: Juergen.ihlemann@llg-ev.de
}

\begin{abstract}
Structured excimer laser back side irradiation of thin $\mathrm{SiO}_{\mathrm{x}}$-films on fused silica substrates is used for the formation of complex micro- and nano-patterns. A polymeric superstrate serving as a confinement layer on top of the $\mathrm{SiO}_{\mathrm{x}}$-film allows the formation of very smooth and regular patterns. Depending on laser processing parameters, either arrays of blisters or cups, or grids with free standing wires below $100 \mathrm{~nm}$ in diameter can be fabricated. To obtain these nano-features with high structure resolution on a comparatively large area with a single KrF-laser pulse (wavelength 248 $\mathrm{nm}$ ), an optical system combining a beam homogenizer with a high resolution mask imaging optical system has been established. At fluences in the range of 300 to $400 \mathrm{~mJ} / \mathrm{cm}^{2}$ very regular grid patterns with wires detached from the substrate and nodes attached to the substrate are obtained. The free standing wires have diameters down to less than $100 \mathrm{~nm}$. The patterned $\mathrm{SiO}_{\mathrm{x}}$-material can be oxidized to $\mathrm{SiO}_{2}$ by thermal or laser treatment.
\end{abstract}

DOI: $10.2961 /$ jlmn.2015.02.0009

Keywords: Laser ablation, patterning, $\mathrm{SiO}_{\mathrm{x}}, \mathrm{SiO}_{2}$, confinement

\section{Introduction}

Silicon suboxide $\left(\mathrm{SiO}_{\mathrm{x}}\right.$ with $\left.\mathrm{x}<2\right)$ has a number of applications in optics and microelectronics. It is used for instance for multilayer coatings in the infrared range [1], for antireflective layers, or as protective coating against corrosion. In electronics, $\mathrm{SiO}_{\mathrm{x}}$ is used e.g. as a dielectric material for thin film capacitors [2]. Furthermore, $\mathrm{SiO}_{\mathrm{x}}$ is used as the source material for the generation of Si-nanocrystals to be used as light emitters in the field of silicon nanophotonics $[3,4]$. For example, by depositing $\mathrm{SiO}_{\mathrm{x}}$ layers just a few $\mathrm{nm}$ thin and heating them in inert atmosphere, material segregation leads to the formation of Si-nanocrystals of a well-defined size [5].

In contrast to $\mathrm{SiO}_{2}$ (quartz or fused silica), $\mathrm{SiO}_{\mathrm{x}}$ is strongly UV-absorbing, thus permitting UV laser processing. The absorption coefficient of $\mathrm{SiO}_{\mathrm{x}}$ at $248 \mathrm{~nm}$ is about $2.7 \times 10^{5} \mathrm{~cm}^{-1}$ for $\mathrm{x} \approx 1$ [6]. The convertibility to $\mathrm{SiO}_{2}$ by thermal annealing in air enables the laser based microfabrication of $\mathrm{SiO}_{2}$-components by depositing $\mathrm{SiO}_{x}$, microstructuring by UV-laser ablation, and final oxidation of the product to $\mathrm{SiO}_{2}$ [7]. In this manner various optical elements like phase masks or complex diffractive optical elements have been fabricated [8]. Furthermore, laser induced forward transfer of $\mathrm{SiO}_{\mathbf{x}}$-films has been demonstrated [9]. Besides these subtractive and additive manufacturing methods, recently a shaping process has been demonstrated starting from thin $\mathrm{SiO}_{\mathrm{x}}$-films and ending in complex wire grids [10]. Polymeric wire grids with similar dimensions made by two photon polymerisation (TPP) have been tested as scaffolds for controlled three dimensional cell culture [11] or cell force measurements [12]. The fabrication of non-polymeric, inorganic grids with chemical and thermal stability could lead to new application possibilities in the field of biological cell patterning, microfluidics, or sensor technology. However, up to now, these wire grids have been obtained only within rather limited fields. For performing e.g. cell adhesion studies, large, homogeneous processing fields are required. In this paper, a concept and set up for providing large area, high resolution processing of silica wire grids are presented.

\section{Experimental and optical design}

80-90 nm thick $\mathrm{SiO}_{\mathrm{x}}$-films with $\mathrm{x} \approx 1$ were deposited on fused silica substrates by ion beam sputtering (Laseroptik GmbH, Garbsen, Germany) and covered with a 1 $2 \mathrm{~mm}$ thick polydimethylsiloxane (PDMS) confinement layer. The function of this superstrate layer will be discussed later.

Single pulse laser irradiation experiments were carried out using a standard $\mathrm{KrF}$-excimer laser emitting at $248 \mathrm{~nm}$ with pulse duration of about $25 \mathrm{~ns}$. A Cr-on-quartz mask consisting of a periodic array of quadratic openings which cover $25 \%$ of the total area and with pitch ranging from 25 - $70 \mu \mathrm{m}$ defines the beam pattern. The mask was imaged onto the sample with a demagnification of 15:1. The $\mathrm{SiO}_{\mathrm{x}}-$ films were irradiated through the substrate (backside configuration) (Fig. 1).

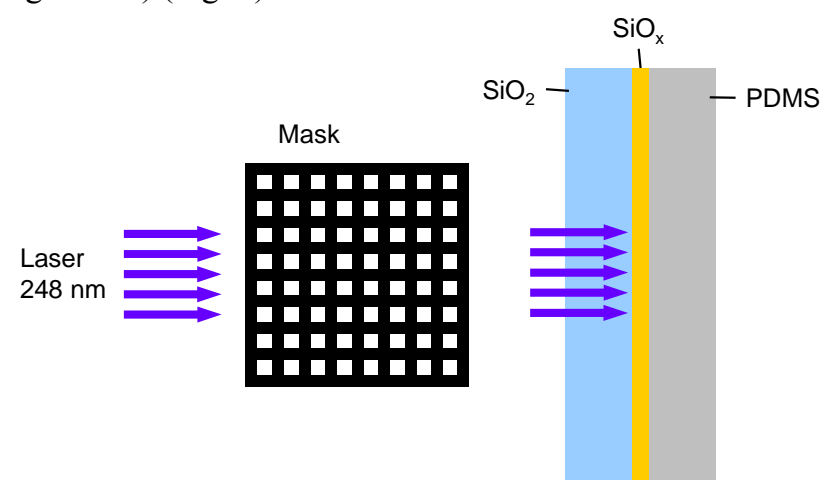

Fig. 1 Scheme of sample irradiation. 


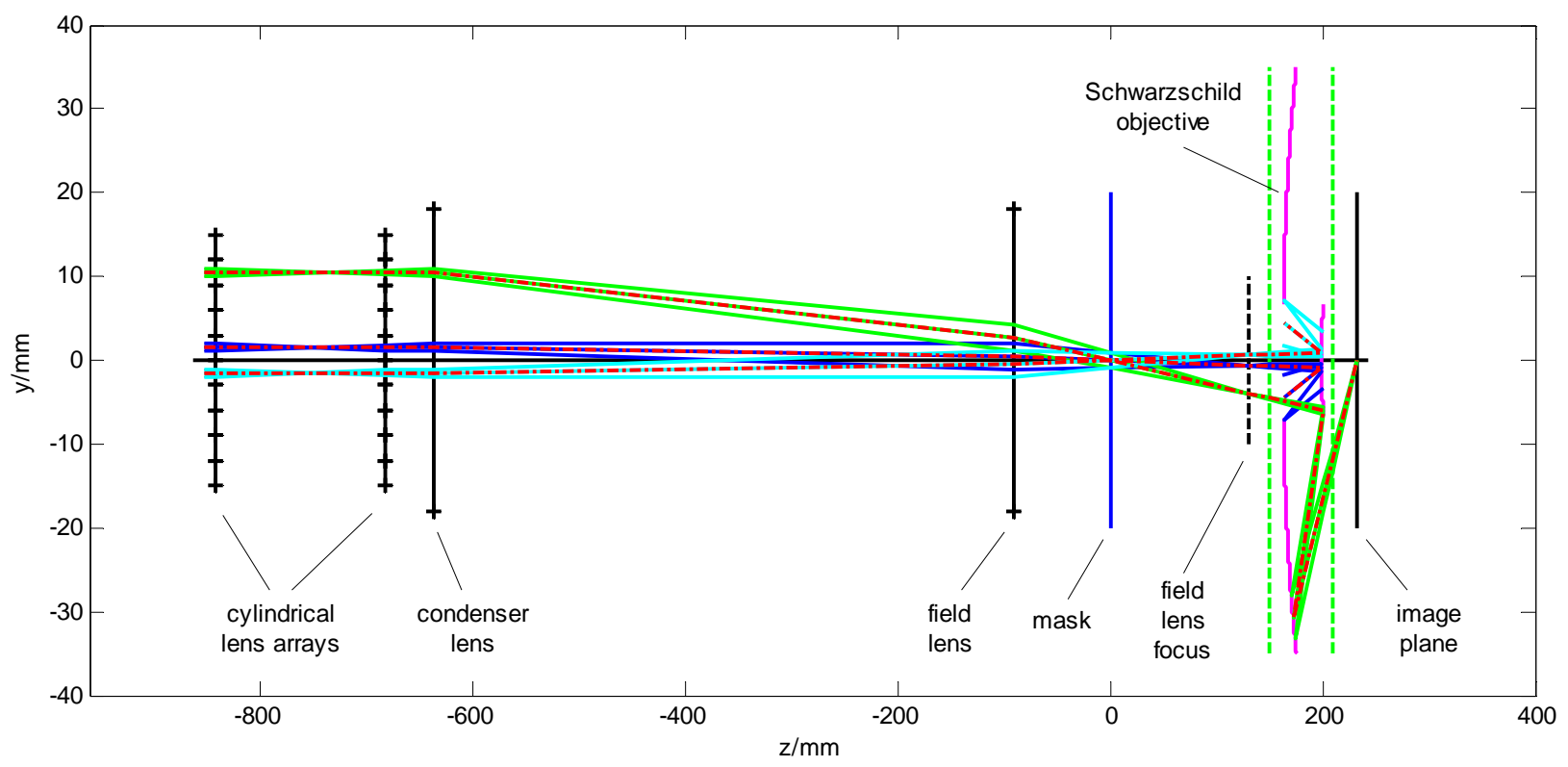

Fig. 2 Optical beam path with beam homogenizer and Schwarzschild type imaging objective.

An efficient and homogeneous mask illumination is provided by a beam shaping system consisting of a cylindrical lens telescope to correct the asymmetric aspect ratio of the laser beam profile followed by a fly's eye condenser in an imaging configuration for beam homogenization. The homogenizer consists of two crossed cylindrical lens arrays segmenting the beam in multiple beamlets and a condenser lens combining the partial beams in the mask plane. It achieves uniform illumination within a field of $6 \times 6 \mathrm{~mm}^{2}$ in the mask plane. To provide high optical resolution, the mask is imaged onto the sample using a Schwarzschild type reflective objective with $15 x$ demagnification yielding a $400 \times 400 \mu \mathrm{m}^{2}$ field of irradiation on the sample. The advantage of using a Schwarzschild objective instead of a refractive lens objective consists in its combination of a high numerical aperture of $\mathrm{NA}=0.5$ with a large working distance of $23 \mathrm{~mm}$.

Condenser lens and field lens (focusing the partial beams before entering the objective) have to be specially arranged to prevent a deterioration of the irradiation homogeneity by partial shadowing of the beam in the Schwarzschild objective. To investigate this effect in more detail, a simple ray tracing simulation of homogenizer and imaging optics has been implemented. Fig. 2 illustrates the optical arrangement including a small number of simulated rays. The cylindrical lens arrays and condenser and field lens are approximated as thin elements. The components have antireflection coatings, so that residual reflections are neglected. The beam intensity is represented by the ray density. The distance between mask and objective is kept fixed to obtain the specified imaging ratio of 15:1. When changing the distance between condenser lens and field lens, the distance between field lens and mask can be regularly adjusted to restore a homogeneous beam in the mask plane. However, this does not always lead to a corresponding homogeneous intensity distribution in the image plane. This behavior is demonstrated in the simulations of the beam profile shown in fig. 3. For the optimum distance between condenser and field lens, the field is homogeneous in the sample plane (fig. 3 (b)). Changing this distance by just 2 $4 \mathrm{~cm}$, a homogeneous field can no longer be obtained. Instead, an area of increased or decreased intensity appears in the center of the spot.

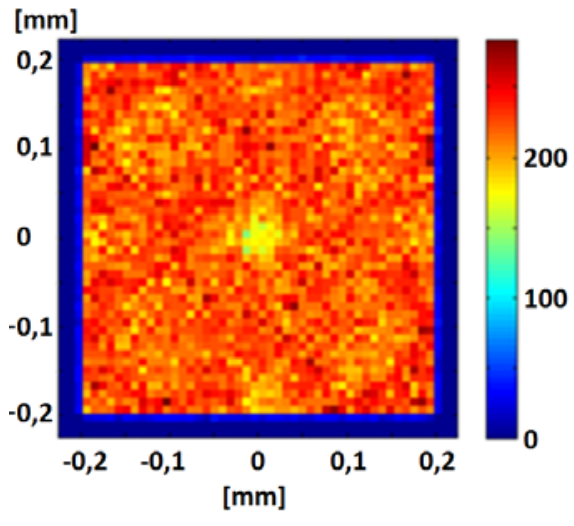

(a) $d=585 \mathrm{~nm}$

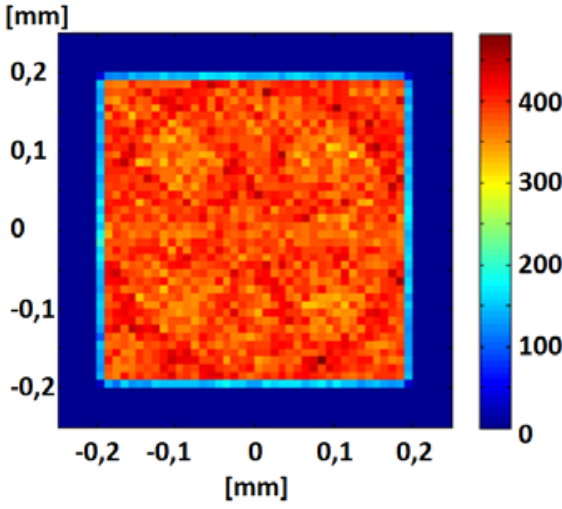

(b) $d=547 \mathrm{~nm}$

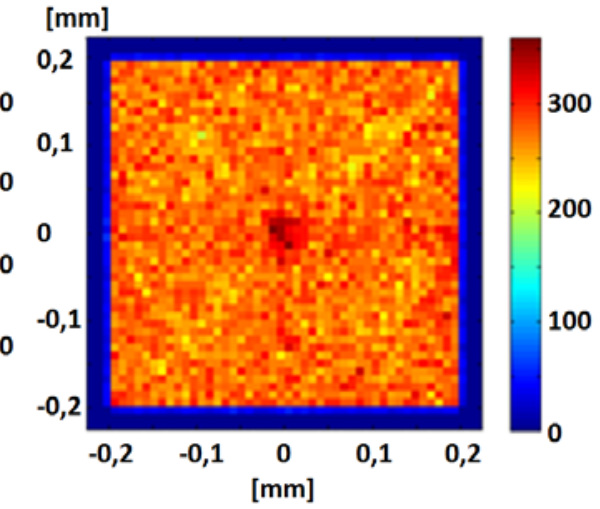

(c) $d=525 \mathrm{~nm}$

Fig. 3 Calculated beam profile in the image plane under variation of the distance $d$ between condenser lens and field lens. 
The effect is caused by partial shadowing of the beamlets close to the optical axis by the annular aperture of the Schwarzschild objective. If, for example, the inner most and most intense beamlets passing the annular aperture are truncated in such a way that the central part of all of them is clipped, the profile in the image will exhibit decreased intensity in the center. The effect of the annular aperture of the objective can also be observed from the simulated beam cross sections along the optical beam path as displayed in fig. 4. As an input to the simulation, a collimated beam with Gaussian profile and beam waist $28 \mathrm{~mm}$ has been assumed as a simplified representation of the pre-shaped excimer laser beam used in the experiments. For the depicted simulation, an optimized configuration of the beam shaping system has been chosen. Only if the superposition of the partly truncated beamlets in the image plane amounts to a constant value, homogeneous sample irradiation is accomplished.

The simulation presented here adopts a simplified view and does neither account for the specific divergence characteristics of the used excimer laser nor for the diffractive beam propagation caused by high resolution mask features. Nevertheless, the results confirm that for each homogenizer configuration (cylindrical lens arrays and condenser) the combination of focal length of the field lens and distance between condenser and field lens has to be optimized.
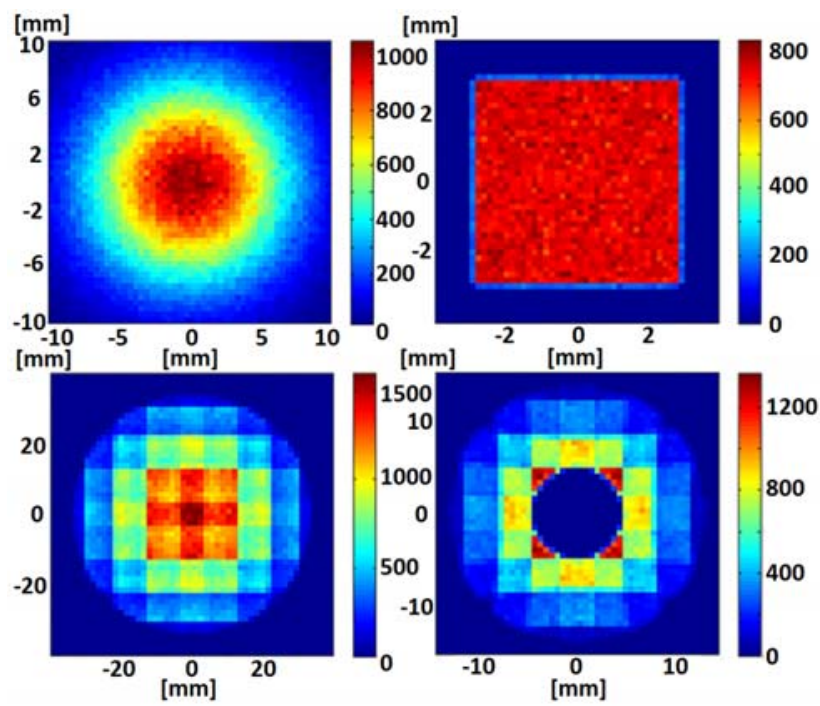

Fig. 4 Calculated beam profile at various positions: input beam (top left), mask plane (top right), primary mirror (bottom left), exit objective (bottom right).

\section{Results and discussion}

Figure 5 shows the influence of the PDMS-superstrate on the irradiation result. Irradiation without PDMS leads to a very irregular pattern of film fragments partially detached from the substrate and partially sticking to it. Irradiation with PDMS on top of the $\mathrm{SiO}_{\mathrm{x}}$-film instead leads to a very regular grid pattern corresponding roughly to the mask pattern. Obviously, the PDMS superstrate acts as a confinement layer serving for shape control and preventing unbalanced disintegration of the $\mathrm{SiO}_{\mathrm{x}}$-film [10]. The close up view in fig. 6 shows that the resulting pattern consists of a network of free standing thin wires connected at nodal points, where this network is fixed to the substrate. Wire diameters of less than $100 \mathrm{~nm}$ are possible.

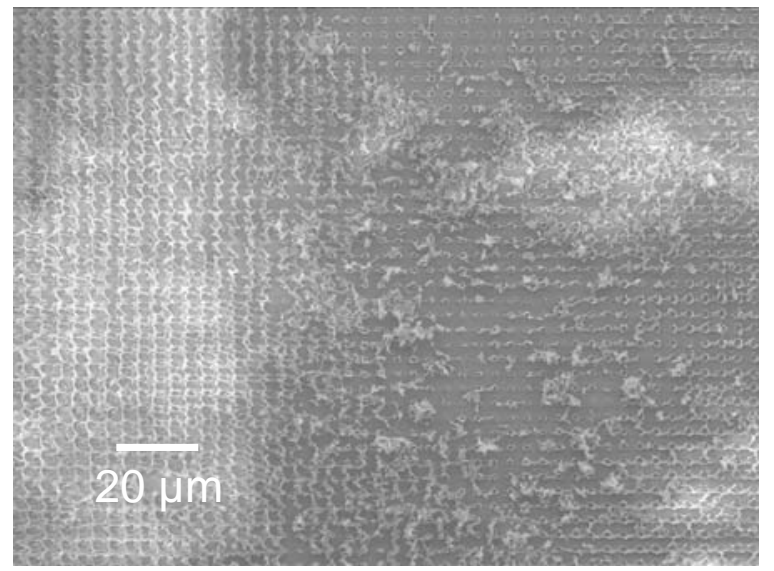

(a)

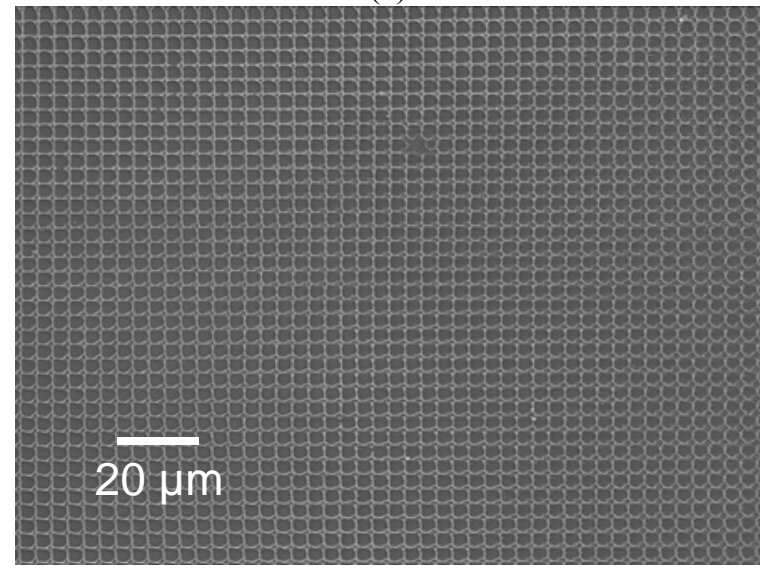

(b)

Fig. 5 Patterned $80 \mathrm{~nm}$ thick $\mathrm{SiO}_{\mathrm{x}}$ film irradiated (a) without and (b) with confinement (after peeling off the confinement layer); laser parameters: $248 \mathrm{~nm}, 380 \mathrm{~mJ} / \mathrm{cm}^{2}$, 1 pulse.

The detachment of the wires from the substrate can be clearly seen in a $45^{\circ}$-view displayed in fig. 7 . In this case, the structure period is $1.7 \mu \mathrm{m}$.

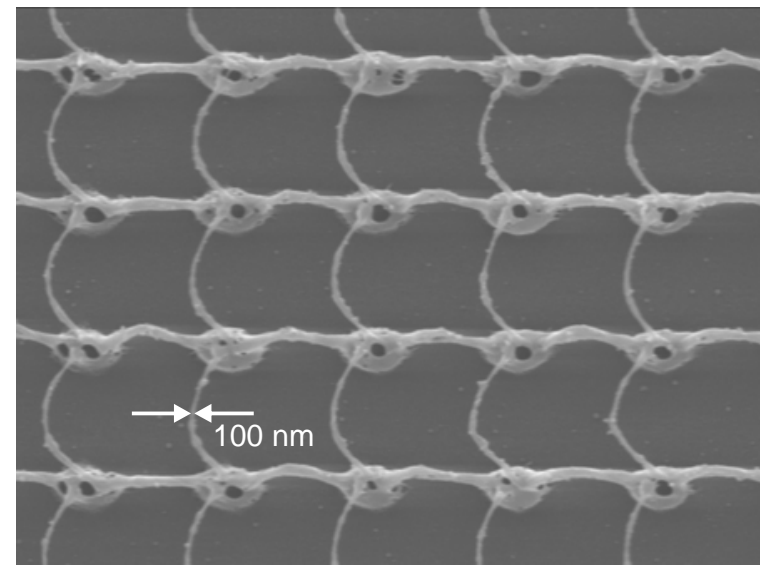

Fig. 6 Patterned $80 \mathrm{~nm}$ thick $\mathrm{SiO}_{\mathrm{x}}$ film irradiated with confinement; laser parameters: $248 \mathrm{~nm}, 380 \mathrm{~mJ} / \mathrm{cm}^{2}, 1$ pulse; structure period: $4 \mu \mathrm{m}$ 


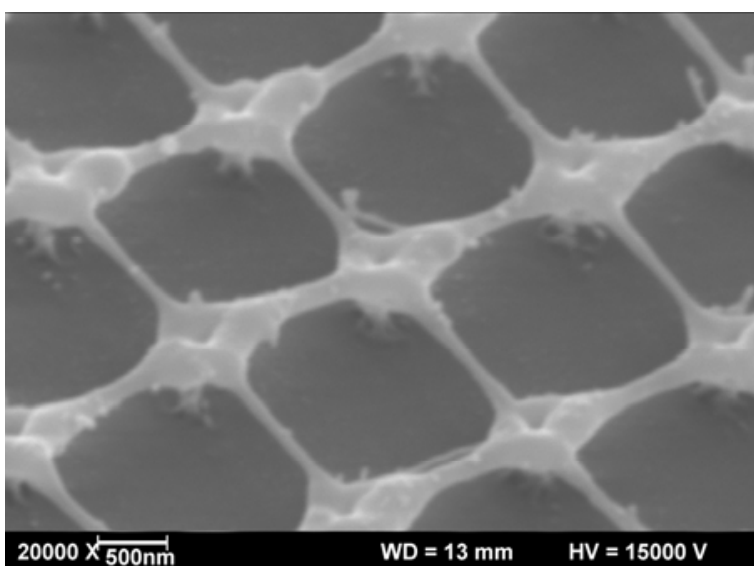

Fig. 7 Patterned $80 \mathrm{~nm}$ thick $\mathrm{SiO}_{\mathrm{x}}$ film irradiated with confinement; laser parameters: $248 \mathrm{~nm}, 350 \mathrm{~mJ} / \mathrm{cm}^{2}, 1$ pulse; structure period: $1.7 \mu \mathrm{m}$.

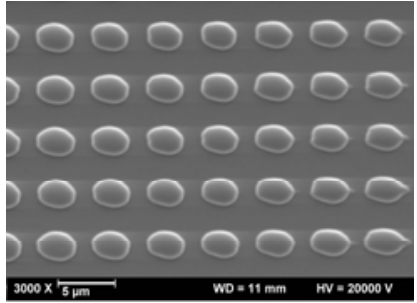

(a)

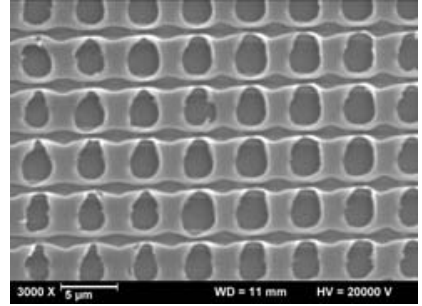

(c)

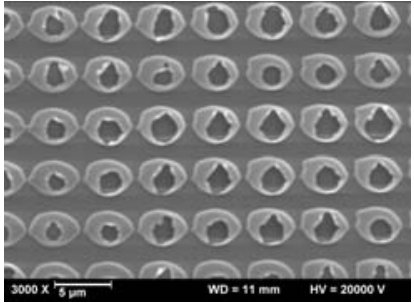

(b)

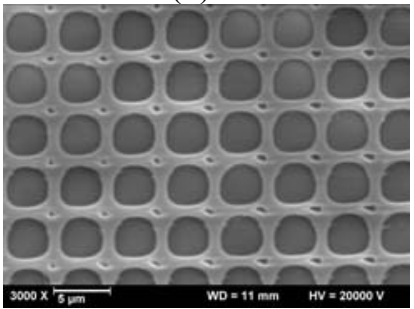

(d)
Fig. 8 Patterned $90 \mathrm{~nm}$ thick $\mathrm{SiO}_{\mathrm{x}}$ film irradiated with confinement; laser parameters: $248 \mathrm{~nm}, 1$ pulse; structure period: $4.7 \mu \mathrm{m}$.

Fluence: (a) $180 \mathrm{~mJ} / \mathrm{cm}^{2}$, (b) $190 \mathrm{~mJ} / \mathrm{cm}^{2}$, (c) $200 \mathrm{~mJ} / \mathrm{cm}^{2}$, (d) $220 \mathrm{~mJ} / \mathrm{cm}^{2}$.

(This film has been deposited by thermal evaporation instead of ion beam sputtering.)

In order to give an impression, how the grid structure may evolve from the irradiation pattern, the results obtained under variation of the fluence are displayed in Figure 8. All fluence data are average values calculated according to pulse energy in the sample plane / total area of the irradiated field. This means that local peak fluences are significantly higher than the stated average values. At low fluence (a), the film detaches from the substrate in the area of the irradiated spots forming blisters. Increasing the fluence, rupture of the blisters and formation of openings in the detached film occurs (b). At still higher fluence the enlargement of the openings and detachment of the film over wide areas are observed $(c, d)$. However, at the posi- tions in between the openings, this grid is still connected to the substrate (small dimples in fig. 8d). This connection to the substrate is conserved, when at still higher fluence the detached film has contracted into a grid of thin wires (cf. fig. 7). Thus, apparently the combination of pulsed laser heating and softening of the thin $\mathrm{SiO}_{\mathrm{x}}$-film, pressure generation leading to expansion damped by the confinement layer, fracture and shaping due to surface tension, and final resolidification lead to the final shape.

Independently of the final shape, these grids can be converted into pure $\mathrm{SiO}_{2}$ by heating to $1200 \mathrm{~K}$ without substantial degradation of the shape [10].

\section{Conclusions}

Grids or other microstructures from chemically stable and biocompatible silica material can be fabricated by single pulse irradiation of a silicon oxide film under polymer confinement. The method may show a route to create surfaces with defined wettability or special cell adhesion properties for microscopy, micro fluidics, or cell culture.

\section{Acknowledgments}

Financial support by the EU and the state of Lower Saxony through EFRE is gratefully acknowledged.

\section{References}

[1] N. Kaiser, H.K. Pulker eds., Optical Interference Coatings, Springer-Verlag Berlin Heidelberg 2003.

[2] P.J. Harrop, D.S. Campbell, Thin Solid Films 2, 213 (1968).

[3] L. Khriachtchev ed., Silicon Nanophotonics, World Scientific Publishing, Singapore 2009.

[4] L. Pavesi, Advances in Optical Technologies, vol. 2008, Article ID 416926.

[5] L. Yi, R. Scholz, M. Zacharias, Journal of Luminescence 122-123, 750 (2007).

[6] M. Jahn, J. Richter, R. Weichenhain-Schriever, J. Meinertz, J. Ihlemann, Appl. Phys. A 2010, 101:533538.

[7] M. Schulz-Ruhtenberg, J. Ihlemann, J. Heber, Appl. Surf. Sci. 248, 190 (2005).

[8] J.-H. Klein-Wiele, J. Békési, P. Simon, J. Ihlemann, Journal of Laser Micro/Nanoengineering 1, 221 (2006).

[9] J. Ihlemann, R.Weichenhain-Schriever, Thin Solid Films 550, 521 (2014).

[10] J. Ihlemann, R. Weichenhain-Schriever, Nanoscale Research Letters 9, 102 (2014).

[11] F. Klein, B. Richter, T. Striebel, C.M. Franz, G. von Freymann, M. Wegener, M. Bastmeyer, Adv. Mater. 23, 1341 (2011).

[12] F. Klein, T. Striebel, J. Fischer, Z. Jiang, C.M. Franz, G. von Freymann, M. Wegener, M. Bastmeyer, Adv. Mater. 22, 868 (2010).

(Received: June 13, 2014, Accepted: February 23, 2015) 